\title{
A MIMO FMCW radar approach to HFSWR
}

\author{
J. O. Hinz and U. Zölzer \\ Department of Signal Processing and Communications, Helmut-Schmidt-Universität/University of the Federal Armed Forces \\ Hamburg, Holstenhofweg 85, 22043 Hamburg, Germany
}

\begin{abstract}
In this paper we propose one possible approach how to apply the concept of multiple-input multipleoutput (MIMO) to monostatic Frequency Modulated Continuous Wave (FMCW) High-Frequency Surface Wave Radar (HFSWR) in a maritime environment. Common tasks for a HFSWR are sea-state monitoring and ship detection, where our focus is on ship detection.

A limiting factor in HFSWR is the available bandwidth, which is inversely proportional to the range resolution capability of the radar and typical below $100 \mathrm{kHz}$. The question is how to extend or combine a conventional single-input multiple-output (SIMO) FMCW phased-array type radar with stretch processing and the colocated MIMO concept to "reuse" the very limited HF radar band resources. Another important question to answer is how MIMO FMCW waveforms can be separated at the receiver.
\end{abstract}

\section{Introduction}

Typical HFSWR systems are used for sea-state monitoring or ship detection, operating in the HF radar frequency band of 3 to $30 \mathrm{MHz}$. These (quasi-)monostatic shore-based systems consist of a "floodlight" transmit antenna with a beamwidth of around $100^{\circ}$ directed at the sea in conjunction with a receiver array of up to 16 elements (Gurgel et al., 1999). Depending on the operating frequency and assuming a receiver antenna element spacing of $\lambda / 2$ the receive array can easily occupy several hundreds meters of coastline. Due to the highly conductive ocean sea surface in combination with a vertical polarization the waves propagate up to few hundreds of kilometres in a so-called ground-wave or surface-wave and experience less attenuation than conventional microwave radars. Due to the low frequency it even allows propagation along the curvature of the earth.

In case of ship detection the worst case is the detection of ships in sea clutter, otherwise detection capability is usually limited by the external noise level. Another important aspect of HFSWR is the high spectrum occupancy by other users, such as radio amateurs or radio stations. Thus it is difficult to obtain a radio frequency interference (RFI) free continuous bandwidth. This is the reason why some authors first monitor the frequency occupancy to select an RFI free or less occupied frequency band (Gurgel et al., 2007) to avoid RFI whenever possible. Other authors propose to improve target detection in sea clutter by using a dual frequency approach (Leong and Ponsford, 2004). Still both approaches will suffer from degraded performance in case the operating frequency does not match the receiver antenna element spacing.

Due to the high dynamic requirements in HFSWR most radar systems use a FMCW waveform in combination with stretch processing to limit the sampling requirements of the analog-to-digital converter (Richards, 2005). This is in contrast to most MIMO approaches where correlation processing is used. This makes it interesting to investigate how a potential MIMO FMCW system could look like and if it is possible to separate the different transmitted signals at the receiver. The rest of the paper will be structured as following. Section 2 is going to introduce the FMCW waveform and the signals at transmitter and receiver as well as stretch processing. After that Sect. 3 gives a deeper insight into HFSWR. Then the paper will proceed with MIMO radar in Sect. 4 and depict the combination of MIMO FMCW and HFSWR in Sect. 5. Finally a conclusion is drawn. 


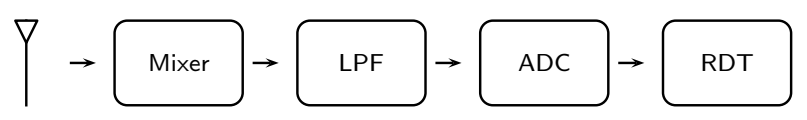

Fig. 1. FMCW receiver structure.

\section{FMCW waveform and Stretch Processing}

The FMCW waveform has three important advantages as compared to a pulse radar system, which are a constant power envelope, a high Doppler shift tolerance and the ability to use a simplified receiver processing called stretch processing. The main disadvantages are the signal leakage from the transmitter into the receiver and range-Doppler coupling. The following equation shows the mathematical description from (Meta et al., 2007) of a single chirp FMCW signal at the transmitter

$s_{t x}(t)=\exp \left(j 2 \pi\left(f_{s} t+\frac{1}{2} \alpha t^{2}\right)\right)$,

in which $t$ is the time-variable within the single chirp period, $f_{s}$ denotes the start frequency of the chirp and $\alpha$ denotes the sweep-rate, which is defined as the ratio of the bandwidth of the chirp $(B)$ divided by the chirp duration $(T)$.

The received signal from a single target is an attenuated, delayed and Doppler-shifted version of the transmitted chirp. In the following only a single non-moving point target is assumed

$s_{r x}(t)=A \cdot \exp \left(j 2 \pi\left(f_{s}(t-\tau)+\frac{1}{2} \alpha(t-\tau)^{2}\right)\right)$,

in which $A$ is used to represent the attenuation factor and $\tau$ is the round-trip time for the signal to propagate from radar system to the target and back. A multiple target case can be seen as a superposition of the different echoes at the receiver.

A typical FMCW single channel receiver structure is illustrated in Fig. 1.

The illustrated mixer in Fig. 1 demodulates the received signal (attenuated, delayed and Doppler-shifted) with the transmitted signal, which is sometimes referred to as reference waveform. This results in a direct demodulation of the received signal into baseband. To eliminate the upper modulation products a low-pass filter is applied. This process is commonly known as stretch processing but sometimes also called deramp processing, deramp on receive, dechirp or onepass processing (Richards, 2005). It is a technique to reduce the sampling-rate requirements of the following analogueto-digital converter (ADC), followed by the range-Doppler transform (RDT). This reduction in sampling-rate is needed due to the high dynamic range. A graphical interpretation of stretch processing for one target can be found in Fig. 2, where the round-trip delay $\tau$ of the receive signal results in a difference frequency $\Delta f$, the so-called beat frequency. Stretch processing can be applied if the target round-trip delay $\tau$ is
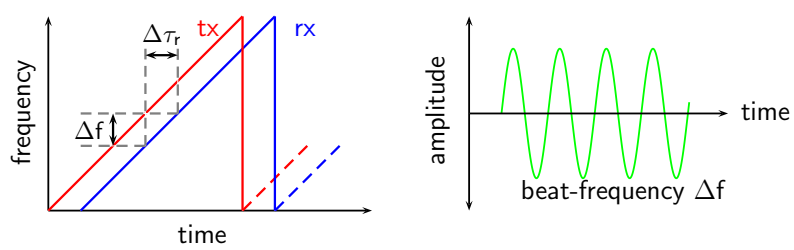

Fig. 2. Stretch processing and resulting beat signal.

small as compared to the chirp duration $T$ and if the beat signal frequency offset due to the delay is large as compared to the Doppler-shift due to the radial velocity of the targets.

In mathematical terms the beat signal of a static point target is expressed in the following equation

$s_{i f}(t)=A \cdot \exp \left(j 2 \pi\left(f_{s} \tau+\alpha t \tau-\frac{1}{2} \alpha \tau^{2}\right)\right)$.

The range resolution $\Delta r$ and Doppler resolution $\Delta f_{D}$ capabilities (McGregor et al., 1994) of the FMCW waveform are given by

$\Delta r=\frac{c}{2 B}$

and

$\Delta f_{D}=\frac{1}{N T}$,

in which the parameter $c$ and $N$ are used to denote the speed of light and the number of chirps considered in one coherent integration time (CIT), respectively. Strongly related to the range resolution and the Doppler resolution is the rangeDoppler transform (RDT). It is a 2-dimensional fast Fourier transform (FFT) used to produce the range-Doppler map (RD map). Sometimes the terms fast time and slow time are used to indicate that the first domain of the transform are the time samples within a single chirp (range transform), while the second domain is across the number of chirps $(N)$ to form the Doppler transform. The purpose of the RDT can be seen as a set of two filter banks used to split the targets according to their range frequency and their Doppler frequency.

\section{HFSWR}

In addition to estimating the range and Doppler shift of a target one is usually also interested in determining the target's azimuth. Due to the limited number of antenna elements and the use of stretch processing in most HFSWRs this task can be performed by digital beamforming as in (Gurgel et al., 1999).

In Fig. 3 the block diagram of a typical SIMO FMCW HFSWR is illustrated. The abbreviation SP following each antenna element is used to indicate stretch processing, thus the combination of mixer and low-pass filtering as described 


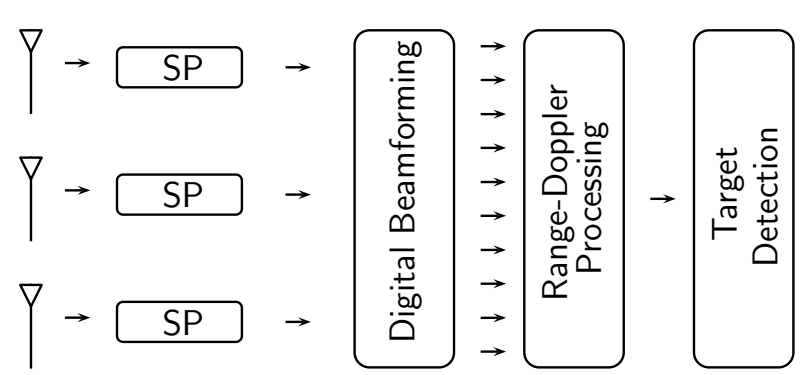

Fig. 3. SIMO FMCW HFSWR receiver structure.

in Sect. 2. The additional bandpass-filters, low-noise amplifiers and ADCs are omitted for simplicity. The low-pass filtered and digitalized baseband antenna elements signals are then fed into a digital beamformer, which in most cases is a Dolph-Chebychev beamformer used to produce a number of formed beams $(\mathrm{M})$ in the angular domain of $-60^{\circ}$ to $+60^{\circ}$. For each of the beams a RDT is applied. The final part is the target detection (TD) in a 2- or 3-dimensional domain.

\section{MIMO}

The term MIMO has become a popular technique in communications and is used to increase the data rate or the reliability of a given link by the use of multiple antennas at the transmitter and the receiver side. Important here is the fact that in this approach all transmitters share the same frequency band, but transmit different signals. It is furthermore required that the receiver is able to separate the signals from each transmit antenna.

For the term MIMO radar one has to distinguish between colocated MIMO radar and statistical MIMO radar. What we consider here is the colocated MIMO radar case, which can be roughly characterized by the condition that the distance between the different transmitter/receiver elements is small as compared to the distance between the radar and the potential targets. The statistical MIMO radar assumes widely spaced transmitter and receiver elements to exploit spatial diversity. Still in both cases each transmitter element is assumed to transmit a different signal, so each receiver element receives a superposition of each transmitter element signal and each target as illustrated in Fig. 4 for the single target case in the far-field.

\section{MIMO FMCW Radar Approach to HFSWR}

The main question is how the concept of colocated MIMO radar can be combined with the linear FMCW waveform and receiver stretch processing in a typical HFSWR environment, possibly even added to an existing SIMO FMCW HFSWR. This implies the selection of appropriate FMCW waveforms to enable the receiver to separate them. In Fig. 5 four possi-

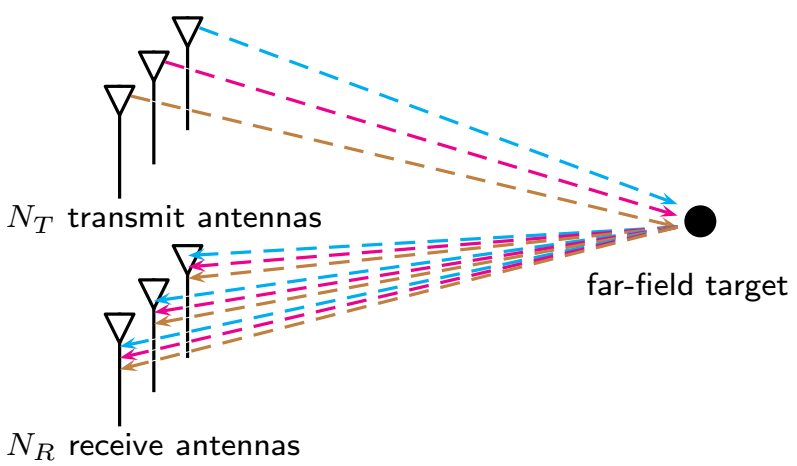

Fig. 4. Colocated MIMO Radar. (a)

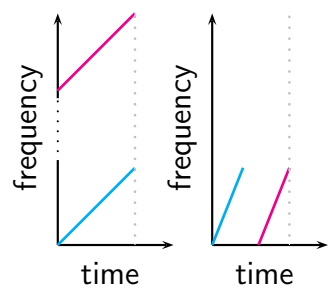

(c)

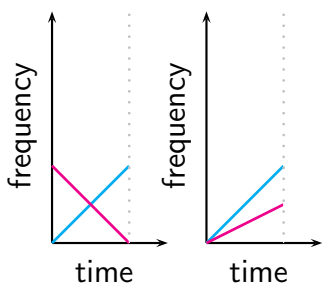

Fig. 5. MIMO FMCW - four possible signalling concepts.

ble signalling concepts (a to d) for two transmit elements are shown.

The two first approaches (a and b) can be summarized as dual-frequency operation (Leong and Ponsford, 2004) and time-staggered operation (Frazer et al., 2007). The first approach enables the receiver to separate the different transmitter FMCW waveforms due to an offset in frequency or in time. The main disadvantage of the dual-frequency operation or even multiple-frequency operation is that with increasing frequency separation between the two transmitted waveform the receiver antenna-element spacing becomes suboptimal for receive beamforming and will either result in a small aperture or the introduction of grating lobes. In addition there are additional licenses required to operate at two or more frequency bands.

The time-staggered approach (b) is an option, where the receiver is able to distinguish the different transmitter elements by their time offset. Still to enable the receiver to distinguish the different waveforms the time offset between the elements has to be larger than the maximum round-trip time $\tau$.

The third option (c) is to use two FMCW waveforms with opposite slope, occupying the same frequency band and having the same time duration. In approach (d) FMCW waveforms with the same start frequency and same duration but different bandwidth and thus different slopes are considered. The RF chain and the antenna element spacing are thus optimal for both last mentioned waveforms. Furthermore no additional licence is required. The main disadvantage of ap- 

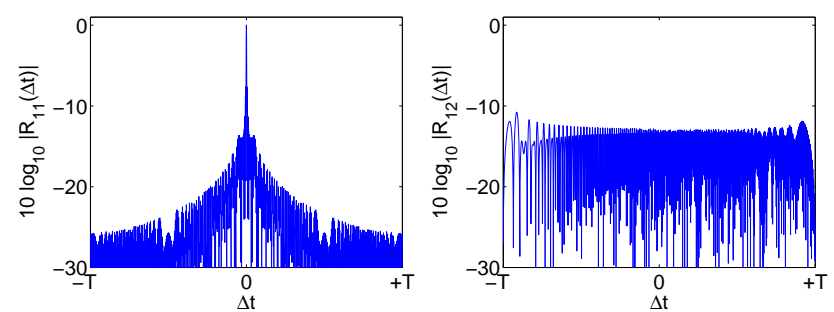

Fig. 6. Normalized auto- and crosscorrelation for opposite slope MIMO FMCW approach (c).

proach (d) is the worsening of the range resolution of the second waveform. Even if in Fig. 5 approach (d) only two different transmit waveforms are shown the approach can easily be extended to more than two waveforms. In the following we are going to investigate the possible application of the MIMO FMCW approach (c) and (d).

\subsection{Correlation properties}

In this section the autocorrelation and crosscorrelation properties of the waveforms are being investigated. The normalized auto- and crosscorrelation of the opposite slope approach (c) are presented in Fig. 6. Here $R_{11}$ is used to denote the normalized autocorrelation of the first waveform while $R_{12}$ indicates the normalized crosscorrelation of waveform one and waveform two. Due to symmetry same applies to the comparison of $R_{22}$ and $R_{21}$, which is not shown here.

Good correlation properties can be characterized by a distinct peak in the autocorrelation function and a low interference level in the crosscorrelation function. In case a correlation-processor based range resolution is used the correlator output with the return of one or several targets will result in one or more distinct peaks.

The detection of these targets is thus limited by the level of noise and clutter as well as additional crosscorrelation residues from other waveforms. In the worst case the additional crosscorrelation will even result in additional peaks in correlation output, which could be misinterpreted as targets. In the presented example of Fig. 6 with two chirps of opposite slopes (c) the signals show good autocorrelation as well as good crosscorrelation properties. Thus the peak of the autocorrelation and average crosscorrelation level differ from each by a level of $14 \mathrm{~dB}$, with no windowing applied. Approach (d) also offers good correlation properties, even if the maximum crosscorrelation level is higher and is not homogeneously distributed. This can be explained by the fact that the instantaneous frequencies over time vary linear in the same direction of higher frequencies. In general the correlation properties are a good indicator if a particular waveform set is suitable for a MIMO approach.

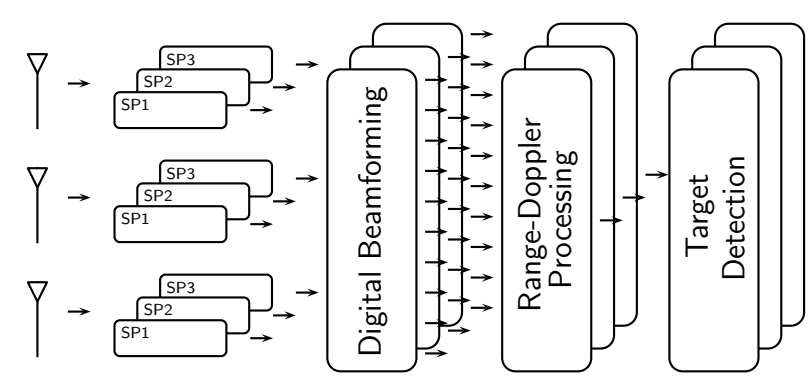

Fig. 7. HFSWR MIMO FMCW receiver structure.
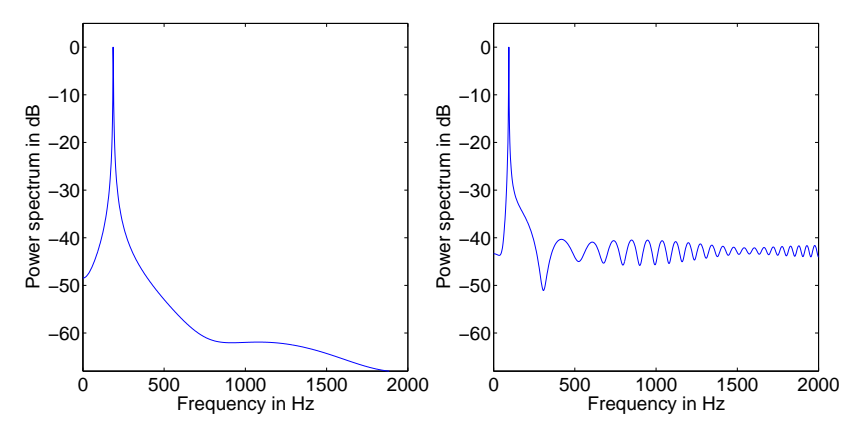

Fig. 8. Stretch processed FMCW for SIMO and MIMO.

\subsection{Proposed receiver structure}

The next step is to investigate if these good properties are remained for the case of stretch processed FMCW. To evaluate this a potential MIMO receiver structure is presented in Fig. 7, which can be seen as an extension of Fig. 3.

Here the abbreviations SP1 to SP $\mathrm{N}_{\mathrm{T}}$ are used to denote stretch processing at each receiver element with the $\mathrm{N}_{\mathrm{T}}$ respective reference transmit waveforms, where in the illustrated example the number $\mathrm{N}_{\mathrm{T}}$ of transmit waveform is chosen to be 3 .

Following the stretch processing are several beamformer, where each one operates across all antenna elements but only takes one particular stretch processed output. After the beamforming the data is range-Doppler transformed, followed by the target detection. Thus the number of receive chains scales with the number of transmit waveforms $\mathrm{N}_{\mathrm{T}}$. This receiver structure is highly motivated by the goal of a potential extension of an existing SIMO system. More advanced receiver concepts might be investigated in the future.

\subsection{SIMO and MIMO stretch processing}

To show the difference in stretch processing of SIMO and MIMO FMCW signals Fig. 8 is presented. It contains the received, demodulated, low-pass filtered and range transformed signal of a single target for the SIMO (only transmitter with $100 \mathrm{kHz}$ bandwidth active) and the MIMO case (transmitters with $100 \mathrm{kHz}$ and $50 \mathrm{kHz}$ bandwidth active) of 
MIMO FMCW approach (d). The formal description of the respective signals can be found as part of Sect. 2. The transmitted waveforms differ by their bandwidth but share the same start frequency $f_{s}$ and the same time duration $T$ of $0.5 \mathrm{~s}$.

If one compares the two parts of Fig. 8 one can see that for the MIMO case the interference level of the additional waveform is increased to a level of $-40 \mathrm{~dB}$. This can be easily explained by the fact that the lower mixing products of two chirps with linear but different slope do not result in one constant frequency difference but in a distribution across the presented frequency range. Still it is clearly possible to determine the characteristic frequency peak of the beat signal of the given target. Same applies if one looks at the other waveform, where the different slope $\alpha$ results in a different beat frequency of the target. Still one has to keep in mind that the range resolution capability of the $50 \mathrm{kHz}$ waveform is worsened as compared to the $100 \mathrm{kHz}$ waveform. By applying range-Doppler transform and target detection independently for both waveforms the target's range and radial velocity can be estimated. Thus both detection results can be used to reassure the existence of a potential target.

\section{Conclusions}

It has been shown that a stretch processed MIMO FMCW approach in HFSWR is possible, in which the transmitter elements operate in the same or partially same frequency band simultaneously and thus the frequency band is reused. This is an important point in HFSWR as a "free" continuous frequency bandwidth is difficult to find.

The presented approach can be seen as an alternative to the dual frequency or the time staggered concepts. Approaches to avoid radio frequency interference and selection of the cleanest frequency can be incorporated into the concept, still limited by the static receiver interelement antenna distance. Additional simulations including attenuation factors and sea clutter and investigation about the allowed number of active transmitters should be performed.

The proposed receiver structure allows the modification of existing SIMO FMCW HFSWR systems and enables the reassurance of a potential target detection. Another modification might be to consider a distributed transmitter system such as a bistatic or multistatic system as the space occupied by an additional "floodlight" transmit antenna is not the limiting factor and is small as compared to the receiver array.

\section{References}

Frazer, G., Abramovich, Y., and Johnson, B.: Spatially Waveform Diverse Radar: Perspectives for High Frequency OTHR, in: IEEE Radar Conference 2007, 385-390, doi:10.1109/RADAR. 2007.374247, 2007.

Gurgel, K. W., Essen, H. H., and Kingsley, S. P.: Highfrequency radars: physical limitations and recent developments, Coastal Engineering, 37, 201-218, doi:10.1016/S0378-3839(99) 00026-5, 1999.

Gurgel, K.-W., Barbin, Y., and Schlick, T.: Radio Frequency Interference Suppression Techniques in FMCW Modulated HF Radars, in: OCEANS 2007 - Europe, 1-4, doi:10.1109/ OCEANSE.2007.4302289, 2007.

Leong, H. and Ponsford, A.: The Advantage of Dual-Frequency Operation in Ship Tracking by HF Surface Wave Radar, in: RADAR 2004 - Toulouse, France, 2004.

McGregor, J., Poulter, E., and Smith, M.: Switching system for single antenna operation of an S-band FMCW radar, IEE Proceedings - Radar, Sonar and Navigation, 141, 241-248, doi: 10.1049/ip-rsn:19941231, 1994.

Meta, A., Hoogeboom, P., and Ligthart, L.: Signal Processing for FMCW SAR, IEEE Transactions on Geoscience and Remote Sensing, 45, 3519-3532, doi:10.1109/TGRS.2007.906140, 2007.

Richards, M. A.: Fundamentals of Radar Signal Processing, McGraw-Hill, 1. edn., 2005. 